\title{
Influence of elastic deformation on the residual ellipticity of polished optical materials
}

\author{
V.P. Maslov, A.Z. Sarsembaeva, F.F. Sizov \\ V. Lashkaryov Institute of Semiconductor Physics, NAS of Ukraine, 41, prospect Nauki, Kiev, Ukraine, \\ Phonelfax:+380442650555,E-mail: sizov@isp.kiev.ua; maslov@isp.kiev.ua
}

\begin{abstract}
The elastic deformation of thin mirrors is widely used in systems of adaptive optics, however, there are no data upon investigations of influence of elastic deformations on parameters of reflected polarised light in the literature. Using the method of ellipsometry, the influence of elastic deformation on the residual ellipticity of polished samples made of optical materials was studied. The results obtained during the researches have shown that the application of elastic deformations leads to essential changes of the minimum ellipticity $\operatorname{tg} \rho$ of polished samples, which testifies to the necessity to take into account this circumstance for devices of adaptive optics, input windows, cryostats and other optical parts working with the changing temperature.
\end{abstract}

Keywords: minimum ellipticity $\operatorname{tg} \rho$, elastic stresses, optical materials.

Paper received 01.10.03; revised manuscript received 27.11.03; accepted for publication 11.12.03.

\section{Introduction}

The elastic deformation of thin mirrors is widely used in systems of adaptive optics, however, in the literature there are no data about investigations of influence of elastic deformations on parameters of reflected polarised light of the object, ellipsometry (reflective polarimetry) is an optical method of measurement. It is based on the analysis of beam polarisation changes of polarised light after its reflection from the studied surface. The ellipsometric method was chosen to control the quality of processing optical surfaces. As a criterion of quality, we selected the minimum ellipticity $\operatorname{tg} \rho[1-3]$.

The main aim of the investigation was to study the influence of elastic deformations on the residual ellipticity of polished samples made of optical materials to make conclusions about the possibilities to use this method to control elastic deformations in optical products.

\section{Method of ellipsometric investigations}

When an electromagnetic wave is leflection from an arbitrary reflecting system (Fig. 1) [2,3], a phase difference appears between components of the electric vector, per- pendicular and parallel to the planes of incidence, which generally leads to the elliptical polarisation of this wave. Reflective indexes $R_{p}$ and $R_{s}$ of a system and phase difference $\Delta$ are by the basic equation of ellipsometry:

$$
\rho=\frac{R_{p}}{R_{S}}=\operatorname{tg} \psi e^{i \Delta} .
$$

Angles $\psi$ и $\Delta$ are called ellipsometric parameters of the system.

The account of multiple reflections inside a layer on the first and second boundary surface allows to express reflective coefficients of all the system, that enter in to the basic ellipsometric equation (1) through the Fresnel reflection coefficients inherent each interface $r_{1}, r_{2}$ and depth of the layer. In this case, equation (1) gets a view:

$\operatorname{tg} \psi e^{i \Delta}=\frac{r_{1 p}+r_{2 p} e^{-i \delta}}{1+r_{1 p} e^{-i \delta}} \cdot \frac{1+r_{1 s} r_{2 s} e^{-i \delta}}{r_{1 s}+r_{2 s} e^{-i \delta}}$

where $\delta=\frac{4 \pi d}{\lambda}\left(n_{1}^{2}-n_{2}^{2} \sin ^{2} \varphi\right)^{1 / 2}-$ phase difference in the layer. 


\section{V.P. Maslov et al.: Influence of elastic deformation on the residual ellipticity...}

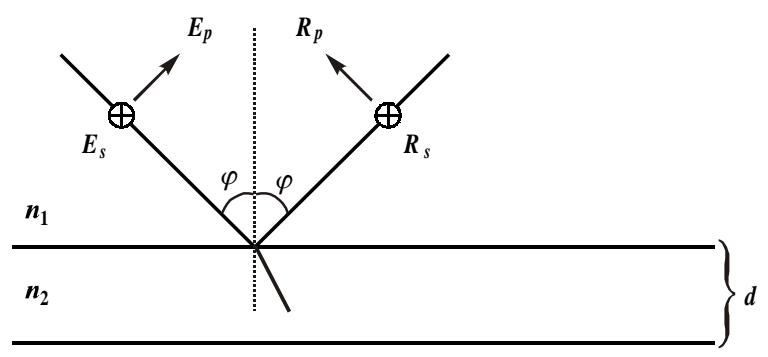

Fig. 1. Reflection of a plane harmonic wave from a homogeneous layer $\left(n_{1}, n_{2}-\right.$ refractive indexes, and $d-$ depth of the sample).

As a result of transformations from (2) the angle dependencies for the phase difference $\Delta$ and angle $\psi$ could be obtained:

$$
\begin{aligned}
& \operatorname{tg}(\Delta-\bar{\Delta})=\frac{4 \pi d\left(n_{1}^{2}-1\right)\left(n_{2}^{2}-n_{1}^{2}\right) n_{2}^{2} \sin \varphi \operatorname{tg} \varphi}{\lambda n_{1}^{2}\left(n_{2}^{2}-1\right)\left(\operatorname{tg}^{2} \varphi-n_{2}^{2}\right)} \\
& \operatorname{tg} \psi=\operatorname{tg} \bar{\psi}\left[1+\frac{4 \pi d\left(n_{1}^{2}-1\right)\left(n_{2}^{2}-n_{1}^{2}\right) n_{2}^{2} \sin \varphi \operatorname{tg} \varphi}{\lambda n_{1}^{2}\left(n_{2}^{2}-1\right)\left(\operatorname{tg}^{2} \varphi-n_{2}^{2}\right)}\right],
\end{aligned}
$$

where $\bar{\psi}$ and $\bar{\Delta}$ - ellipsometric angles for substrate.

At the Brewster angle $\left(\operatorname{tg} \varphi_{\mathrm{Br}}=n_{2} / \mathrm{n}_{1}\right)$ from the second equation of the system (3) the formula for minimum value of ellipticity follows:

$$
\operatorname{tg} \psi=\frac{\pi d\left(n_{1}^{2}-1\right)\left(n_{2}^{2}-n_{1}^{2}\right)}{\lambda n_{1}^{2}\left(n_{2}^{2}-1\right)}\left(n_{2}^{2}+1\right)^{1 / 2}
$$

Relation of Fresnel reflection coefficients of p- and scomponents of the electric vector, $\operatorname{tg} \rho$ and the phase difference between them can be counted by usage of the metaloptics method. The photoelectric method of Beattie and Conn is the most suitable among them. The modification of this method, that allows to apply it to transparent dielectrics was used in this work $[4,5]$.

The directly measured values are intensities of radiation reflected from the sample $I_{0}, I_{45}, I_{90}$, measured at three azimuths of the analyser $\alpha_{a}$ (equal, accordingly, to $0^{\circ}, 45^{\circ}, 90^{\circ}$ ) concerning the plane of incidence and fixed azimuth the polariser $\beta=45^{\circ}$. Ellipsometric parameters are calculated by the formulas:

$$
\begin{aligned}
& \operatorname{tg} \rho=\operatorname{tg} \psi \sqrt{\frac{I_{0}}{I_{90}}} \\
& \cos \Delta=\frac{2 I_{45}-I_{0}-I_{90}}{2 \sqrt{I_{0} I_{90}}}
\end{aligned}
$$

As the measurements of ellipsometric parameters are carried out within the limits of the Brewster angle, where $\cos \Delta$ passes through zero point, the error of phase difference is minimum, and the following condition is realised:

$\operatorname{tg} \rho=\operatorname{tg} \psi$

\section{Experimental results and discussion}

The comparative estimation of deformation influence of substrates made of different materials on the ellipsometric parameters (EP) was performed. The researches were made for optical glass K8 (analogue BK7), silica glass $\mathrm{KB}$ and sitall CO115M (analogous to ZERODUR). Selection of materials for these samples was based on such facts: glass and glassceramic are model samples and they have practical use in instrument making, particularly for manufacturing adaptive mirrors. $25 \mathrm{~mm}$ diameter samples were previously polished by electroemery with stippling M28 and M10, and then glazed with polirit on the depth of $30 \mathrm{~m}$. Thickness of samples was $2 \mathrm{~mm}$. Flank surfaces of samples were made with angle $3^{\circ}$ to the polished surface and was blackened with a varnish. Samples were designed with the requirement on rejection from planes:

$N=1 \ldots 5$

$\Delta N=0,5 \ldots 1$

where $N$ - interferential Newton rings; $\Delta N$ - rejection of interferential rings [6].

The laser null-ellipsometer LEF-3M-1 $(\lambda=6328 \AA)$ was used for the measurements.

It is known, that the value of ellipticity decreases with the increase of polishing depth down to $8-10 \mu \mathrm{m}$, and with further increase of the depth becomes stabilised, remaining a constant for deep-polished samples [2]. Such nature of ellipticity changes is explained by decreasing of defects concentration with increase of polishing depth. Thus, there was a vague role of residual stresses, which appears as the result of mechanical operation.

Studying of EP changes in surface layers of samples was conducted using the special device (Fig. 2).

Samples were pasted by the blackened surface to the frame 1 of the device. Using the abutment screw tensile 2, the elastic deformation was created on the surface of a polished sample. Sample flatness changed.

The control of sample deformation was conducted using a tentative glass for observation of interferential Newton rings in the air interspace between the studied sample and tentative glass. $5 \mu \mathrm{m}$ deformation responded

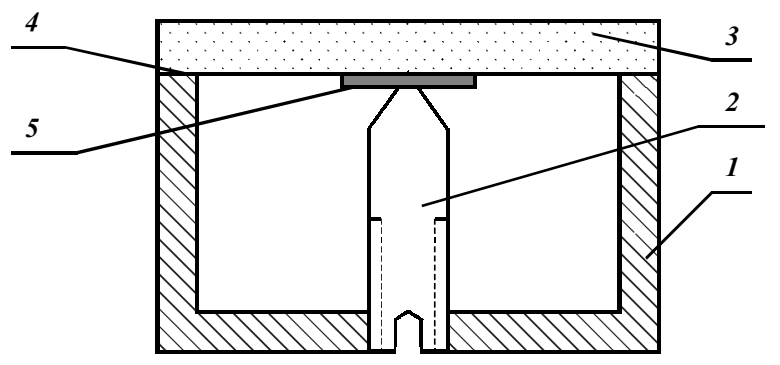

Fig. 2. Device for the deformation of surfaces of polished samples: 1 - the frame of the device, 2 - the screw, 3 - the sample, 4 - glue, 5 - the paper gasket. 
V.P. Maslov et al.: Influence of elastic deformation on the residual ellipticity...

Table 1. Dependence of ellipsometric parameters of sitall CO115M, silica glass KB, glass K8 on their deformation. tg $\rho$ - minimum ellipticity, $\Phi^{\circ}$ - main angle.

\begin{tabular}{|l|c|c|c|c|c|c|c|}
\hline \hline \multicolumn{2}{|c|}{ Deformation } & \multicolumn{2}{c|}{ Sitall CO115M } & \multicolumn{2}{c|}{ Silica glass KB } & \multicolumn{2}{c|}{ Glass K8 } \\
\hline$\mu \mathrm{m}$ & $\begin{array}{l}\text { Number of } \\
\text { interf. rings }\end{array}$ & $\operatorname{tg} \rho \cdot 10^{-3}$ & $\Phi$, (degrees) & $\operatorname{tg} \rho \cdot 10^{-3}$ & $\Phi$, (degrees) & $\operatorname{tg} \rho \cdot 10^{-3}$ & $\Phi,($ degrees) \\
\hline 0 & 0 & $3.5 \pm 0.4$ & $57^{\circ} 12^{\prime} \pm 3$ & $3.50 \pm 0.4$ & $55^{\circ} 33^{\prime} \pm 3$ & $4.3 \pm 0.4$ & $56^{\circ} 41^{\prime} \pm 3$ \\
\hline 2.5 & 10 & $4.1 \pm 0.4$ & $57^{\circ} 13^{\prime} \pm 3$ & $4.16 \pm 0.4$ & $55^{\circ} 39^{\prime} \pm 3$ & $4.5 \pm 0.4$ & $56^{\circ} 41^{\prime} \pm 3$ \\
\hline 5 & 20 & $5.0 \pm 0.5$ & $57^{\circ} 18^{\prime} \pm 3$ & $4.46 \pm 0.5$ & $55^{\circ} 45^{\prime} \pm 3$ & $4.8 \pm 0.5$ & $56^{\circ} 42^{\prime} \pm 3$ \\
\hline $\begin{array}{l}\text { After deformation } \\
\text { removing }\end{array}$ & $3.6 \pm 0.1$ & $57^{\circ} 11^{\prime} \pm 3$ & $3.33 \pm 0.1$ & $55^{\circ} 38^{\prime} \pm 3$ & $4.26 \pm 0.1$ & $56^{\circ} 42^{\prime} \pm 3$ \\
\hline $\begin{array}{l}\text { Relative shift of } \\
\text { tgc at deformation }\end{array}$ & $42.8 \%$ & - & $31.4 \%$ & - & $11,6 \%$ & - \\
\hline
\end{tabular}

a crimp of the studied sample up to 20 interferential rings. The increase of the load till the appearance of the greater number of Newton rings can cause to the appearance of microcracks and exceeded material stability in conditions of the central crimp caused by the pressure (80100) $\mathrm{MPa}$. Thus, the applied stresses and deformations up to $5 \mathrm{~mm}$ were with the elastic, range.

In course of experiments, the statistical method of analysing the results was used: the random component of the error limit at the confidence coefficient 0.95 was equal to $10 \%$ from the value of EP.

The results are shown in the Table 1 .

During deformation of the polished surface of sitall, the main angle $\Phi$ a little bit increases and returns to the initial value after removing the deformation. The ellipticity at deformation is considerably increased and returns to initial value after removing the deformation.

For polished surface of silica glass, the main angle before deformation was less then the Brewster one. Its increase with loading can testify the approach of matter properties to the bulk ones. It is related with relaxation of defects caused by the mechanical treatment at a padding influence of bending external stresses.

For polished surface of the glass $\mathrm{K} 8$, the main angle is less than the Brewster angle. When applying the distorting efforts and their following removal the main angle changes a little. The ellipticity changes similarly to those of sitall and silica glass.

The obtained data have shown, that there is a relative shift of tgc for studied samples. The largest shift is for sitall, and the smallest one is for the glass $\mathrm{K} 8$. In our opinion, it is related with a bulk structure of the matter: the minimum ellipticity shift will correspond to the most homogenous matter.

It is necessary to mark such experimental fact, that after removing of external stress the dispersion of ellipsometric parameters of studied samples for the above mentioned materials become smaller than before deformation.

\section{Conclusions}

The application of incurvating elastic deformations, of which value approximates to destructive values, leads to the essential changes of the minimum ellipticity $\operatorname{tg} \rho$ for the polished samples made of sitall CO115M, silica glass $\mathrm{KB}$ and glass $\mathrm{K} 8$. This is related with a bulk structure of the matter. The main angle $\Phi$ practically does not change. The returning of tgc practically to initial values after removing the load is observed. The relative error of experimental results of minimum ellipticity tgc researchers decreases, which can testify to an increasing homogeneity of upper layers of the studied materials after applying of mechanical loads in the area of elastic deformations. So it's possible to use this method to control the elastic deformations in optical products.

\section{References}

1. T.V. Vladimirova, N.Ya. Gorban, V.P. Maslov, T.S. Melnik, V.A. Odarich, The research of the optical proper ties and building of sitall // OMP, 1979, №9, p.31-33;

2. Fundamentals of an ellipsometry. Under edition of A.V. Rzhanov // Novosibirsk, Nauka, 1979;

3. T.V. Andreeva, V.A. Tolmachev, Methodological aspects of ellipsometric experiment on optical materials // OMP, 1986, №10, p. 36-39;

4. V.A. Odarich, Measurement of small ellipsometric parameters by a photoelectric method // Zav.lab., 1977, №43, p.1093;

5. Beattie G.R, Conn G.K.T., Phil. Mag., 1955, 46, p. 222-225;

6. The reference book of the technologist - optician, under edition of S.M. Kuznezcova, M.A. Okatova, Leningrad, Magnitostroenie, Leningrad department, 1983, 414 pages;

7. A.I. Belyaeva, A.A. Galuza, T.G. Grebennik, V. Pyuriyev, Optical constants of surface layer on gadolinium gallium garnet: ellipsometric study // Semiconductor Physics, Quantum Electronics and Optoelectronics, 1999, 2(4), p. 61-65. 